\title{
Performance of Pattern Recognition Myoelectric Control Using a Generic Electrode Grid with Targeted Muscle Reinnervation Patients
}

\author{
D.C. Tkach, A.J. Young, Student Member, IEEE, L.H. Smith and L.J. Hargrove, Member, IEEE
}

\begin{abstract}
Targeted muscle reinnervation (TMR) is a surgical technique that creates myoelectric prosthesis control sites for high-level amputees. The electromyographic signal patterns provided by the reinnervated muscles are well-suited for pattern recognition (PR) control. PR control uses more electrodes compared to conventional amplitude control techniques but their placement on the residual limb is less critical than for conventional amplitude control. In this contribution, we demonstrate that classification error and realtime control performances using a generically placed electrode grid were equivalent or superior to the performance when using targeted electrode placements on two transhumeral amputee subjects with TMR. When using a grid electrode layout, subjects were able to complete actions $0.290 \mathrm{sec}$ to $1 \mathrm{sec}$ faster and with greater accuracy as compared to clinically localized electrode placement (mean classification error of $1.35 \%$ and $3.2 \%$, respectively, for a 5 movement-class classifier).These findings indicate that a grid electrode arrangement has the potential to improve control of a myoelectric prosthesis while reducing the time and effort associated with fitting the prosthesis due to clinical localization of control sites on amputee patients.
\end{abstract}

\section{INTRODUCTION}

Major upper-limb amputation is a significant cause of disability in the US that affects over 41,000 individuals [1]. Currently, prosthetic devices are the most effective means of treatment. The advent of myoelectric prostheses provided amputees with more intuitive means of controlling their artificial limb than with traditional body-powered prostheses. This capability is achieved by translating electromyographic (EMG) signals from residual-limb muscles into motor prosthesis commands. Typically, the amplitude of the EMG signal recorded from an appropriate muscle site is used to directly control the actuator of the corresponding joint in the

A. J. Young was supported by the National Science Foundation and NDSEG Graduate Research Fellowship 32CFR168a. L. H. Smith was supported by a Howard Hughes Medical Institute Research Fellowship.

D. C. Tkach is with the Center for Bionic Medicine at the Rehabilitation Institute of Chicago, Chicago, IL 60611 USA (e-mail: dtkach@ric.org).

A.J. Young is with the Center for Bionic Medicine at the Rehabilitation Institute of Chicago, Chicago, IL 60611 USA and with the Department of Biomedical Engineering at Northwestern University (e-mail: ajyoung@u.northwestern.edu).

L.H. Smith is with the Center for Bionic Medicine at the Rehabilitation Institute of Chicago, Chicago, IL 60611 USA and with the Department of Biomedical Engineering at Northwestern University (e-mail: laurensmith@fsm.northwestern.edu).

L. J. Hargrove is with Center for Bionic Medicine at the Rehabilitation Institute of Chicago, Chicago, IL 60611 USA and with the Department of Physical Medicine and Rehabilitation at Northwestern University, Chicago, IL, 60611, USA (e-mail: 1-hargrove@northwestern.edu). prosthesis[2]. Although this type of "direct" control has gained clinical acceptance, it is fraught with some significant shortcomings. Due to limited independence of EMG signal sources, the practical capability of "direct" control remains limited to 2 degrees of freedom [3]. Additionally, "direct" control is neither naturalistic or intuitive in some circumstances, as it requires that users avoid co-contracting their muscles when they wish to achieve smooth control of the prosthesis and then actively co-contract in order to switch control between the available degrees of freedom.

Two major advances in the field have provided the means of achieving more intuitive control of a myoelectric prosthesis. The first is the Targeted Muscle Reinnervation (TMR) surgical procedure which transfers the nerves from an amputated limb to biomechanically non-functional muscles. The reinnervated muscle then becomes a biological amplifier of the nerves from the amputated limb and provides a convenient site for EMG recording [4]. A key benefit of TMR is that it offers the means for intuitive, physiologically appropriate simultaneous control of multiple degree of freedom prostheses while using "direct" control scheme as described earlier. The other major advancement in the field is the adoption of alternative prosthesis control algorithms such as pattern recognition $[5,6]$. Pattern recognition predicts desired limb motion based on the patterns embedded within the EMG signals recorded from multiple sites on the amputee's residual limb. The use of pattern recognition with TMR subjects provides accurate and naturalistic control of a myoelectric prosthesis for high-level, upper-limb amputee patients [4].

In contrast to pattern recognition control, conventional "direct" - EMG control of myoelectric prosthesis requires precise placement of bipolar electrode pairs over the reinnervated muscle sites, such that the measured EMG has very little muscle crosstalk. Pattern recognition controllers, however, do not require that the EMG signals be free from cross-talk [7]. The pattern recognition algorithm simply requires a set of unique EMG patterns for classification of each motion. As such, the physical location, or source, of the pattern is irrelevant as long as the patterns are unique [8]. Evaluating the number of EMG channels and their locations has been previously investigated for transradial amputees [9] and TMR patients [10] using high-density electromyography with channel reduction. These previous results from TMR patients suggest that there is a significant benefit of strategic placement of electrodes over the reinnervated muscle sites when up to 16 movements are recognized by the classifier. It is, however, unclear whether this holds true if fewer motions are controlled. 
In this study, we compared the effect of using a generic, array-type electrode placement to conventional, precise electrode placement on the performance of pattern recognition-based prostheses control by transhumeral amputees with TMR in a virtual environment.

\section{METHODS}

\section{A. Data Collection}

Surface EMG recordings were collected from two transhumeral amputee subjects - subjects TH1 and TH2. Both subjects have undergone TMR surgery following their initial amputation procedure. The long head of the biceps and the long head of the triceps remained normally innervated, whereas the short head of the biceps and the lateral head of the triceps were reinnervated. This yielded four total direct control sites (one normally innervated and one reinnervated site on both the biceps and triceps) for each subject. $\mathrm{Ag} / \mathrm{AgCl}$ surface EMG electrodes (Bio-Medical Instruments) were used to record EMG. The study consisted of two conditions. In the first condition ("DC Site") eight bipolar pairs of EMG electrodes were placed over each of the subject's 4 direct control sites (4 DC Sites) and over 4 additional sites adjacent to those (8 DC Sites) using the localization procedure suggested by Huang et al [10]. Direct control sites were localized by clinical best practices and the inter-electrode distance was $15-30 \mathrm{~mm}$ (Fig. 1a). In condition 2 ("Grid"), 15 individual EMG electrodes were arranged in a grid around the residual limb of each subject as to provide equidistant coverage of the limb (Fig. 1b). The grid was arranged in 3 rings of 5 electrodes. The 5 electrodes in each ring were distributed circumferentially around the residual limb. Each ring was offset relative to the other ring along the long axis of the limb. Spacing between each ring as well as spacing between each electrode within the ring was based on the geometry of the patient's limb and computed to provide equidistant placement of electrodes. Grid electrodes were paired to create 15 bipolar EMG channels. Pairings were organized along the long axis of the residual limb, connecting electrodes on adjacent rings (ring 1 electrodes connected to ring 2 electrodes and ring 2 electrodes, connected to ring 3 electrodes) with interelectrode distances ranging 30-60 mm. 10 electrode pairings were made in this manner. An additional 5 pairings were created by connecting electrode sites that were diagonally offset from each other (Fig. 1b) with inter-electrode distances ranging $80-100 \mathrm{~mm}$.

Training and testing data were recorded for nine movement classes: wrist pronation, wrist supination, wrist flexion, wrist extension, hand open, hand close, elbow flexion, elbow extension, and a relaxed (or no motion) class. Subjects performed contractions for $3 \mathrm{~s}$ in each of eight repetitions, producing a total of $12 \mathrm{~s}$ of training data and $12 \mathrm{~s}$ of testing data per movement class with each of the two electrode configurations.

EMG signals were preprocessed by amplification and high-pass filtering $(20 \mathrm{~Hz}$ cutoff frequency). Data were segmented into $250 \mathrm{~ms}$ windows with $50 \mathrm{~ms}$ of overlap [11]. TD (Time Domain) and AR (Autoregressive) features were extracted from the EMG signals. The TD feature set included mean absolute value, zero crossings, slope sign changes, and waveform length. The AR feature set included the six coefficients of a 6th order autoregressive model, which was selected based on previous related work [12]. Linear discriminant analysis (LDA) was used for pattern recognition control (feature classification) because of its computational efficiency and its accuracy is comparable to other classification techniques. A 9 movement-class (MC) classifier (all movements), a $7 \mathrm{MC}$ classifier (excluding wrist pronation and supination) and a $5 \mathrm{MC}$ classifier (excluding wrist pronation, supination, flexion and extension) were trained using recorded data.

We used a Target Achievement Control (TAC) test to evaluate real-time control performance of subjects using the five-class classifier. The TAC test prompts the user to move a virtual hand to designated postures while providing realtime visual feedback of the virtual arm's position. Full description of this test is provided elsewhere [13]. In this study, each prompted posture required the user to perform two sequential motion classes, but all five classes were active at all times. Each target posture required subjects to move the virtual prosthesis through $75^{\circ}$ of motion for both DOF into a target zone designated by a grey outline of a virtual limb. Once there, the virtual limb changed color, instructing subjects to maintain the prompted posture within the target zone. For successful completion of a trial, subjects had to reach the prompted posture with a 20 second time window and maintain that posture within a $30^{\circ}$ tolerance for 1 second. During the TAC tests, we imposed a decisionbased velocity ramp on our controller to minimize the effect of unintended movements. The ramp functions by attenuating movement speed following a change in classifier decision[14]. One test consisted of 4 repetitions for each possible target posture. One TAC test was performed with electrodes arranged in the grid configuration and one TAC test was performed with electrodes placed over reinnervated, direct control sites.
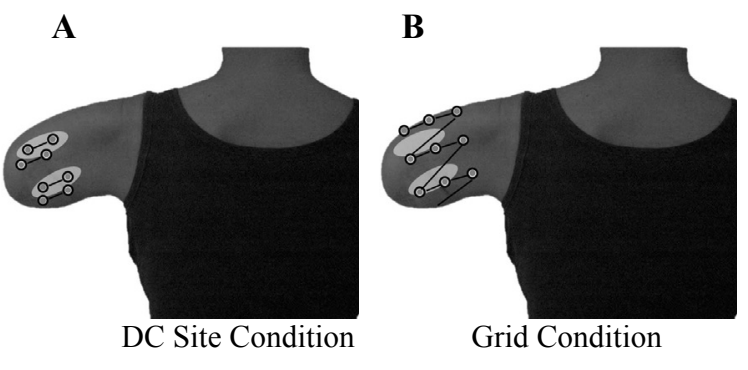

Fig.1 Posterior view of electrode placement for (a) clinical configuration targeting reinnervated, direct control sites (experimental condition "DC Site") and (b) grid placement (experimental condition "Grid"). Solid line connections represent differential bipolar electrode pairs, and lighter ovals represent clinical direct control sites. In the DC Site configuration (a), 8 bipolar pairs of adhesive electrodes were placed at 4 direct control sites. In the Grid configuration (b), 15 adhesive electrodes were evenly spaced in a $5 \times 3$ grid around the circumference of the residual limb without regard to direct control sites. Fifteen differential bipolar pairs were used: 10 oriented axially along the length of residual limb, 5 oriented obliquely.

\section{B. Data Analysis}


TAC test performance was evaluated using two measures: completion rate and completion time. Completion rate is defined as the percentage of trials successfully completed and completion time is defined as the average time across all motions to complete a trial. We thus assess controllability of the virtual prosthesis by evaluating the completion rate as a function of completion time.

In addition to evaluating TAC test performance we also evaluated LDA classification error of the $5 \mathrm{MC}$ classifier as well as 7 and 9 MC classifiers. Four fold cross validation was used to evaluate offline classification error.

Lastly, we performed sequential forward selection (SFS) to determine the optimal number of EMG channels in an electrode grid (i.e. grid size). In this analysis, classification error was assessed for each type of the classifier (5, 7 and 9 MC classifiers) as the size of the set of EMG channels used by the classifier varied from 1 channel to 15 channels.

\section{RESULTS}

\section{A. Real time performance of virtual prosthesis control}

Both subjects achieved $100 \%$ trial completion rate for the TAC test. This is not unexpected as both subjects are experienced users of myoelectric prostheses and are also experienced users of pattern recognition control. However, subject TH2 was able to reach prompted postures faster (1 second faster on average) when EMG electrodes were arranged in a grid pattern as compared to when EMG electrodes were positioned over the reinnervated, direct control sites (Fig. 2). Both subjects TH1 and TH2 reached the prompted postures faster when EMG electrodes were arranged in a grid configuration (290ms and 1s faster on average, respectively). Statistical analyses were not made due to the small sample size.

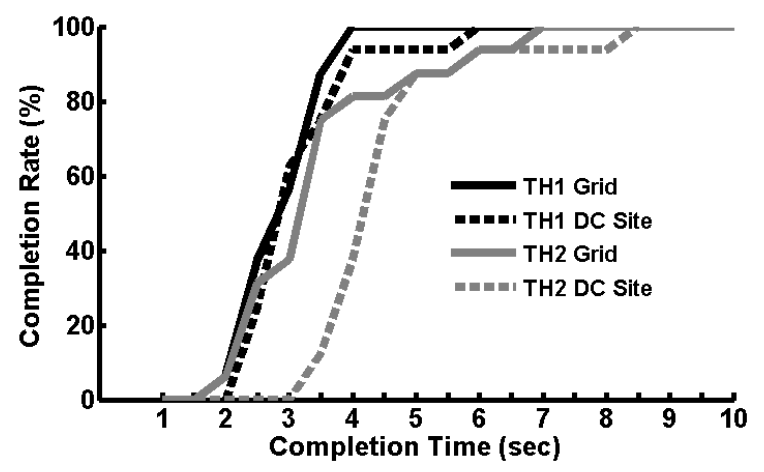

Fig.2 Completion rate curves for the two conditions. Solid line indicates performance during condition 2 (Grid) trials and dashed lines indicate performance during condition 1 (DC Sites). Lines in black show subject TH1 data and lines in grey show subject TH2 data. The data shown represents pooled completion rates and times from all possible postures and repetitions.

\section{B. Classification error for 5, 7 and 9 MC classifiers}

Consistent with the trend seen in the controllability of the virtual prosthesis, the classification error of the $5 \mathrm{MC}$ classifier used during the TAC test for subject TH1 was lowest when EMG signals were acquired from electrodes arranged in a grid pattern as compared to electrodes placed over direct control sites (Fig. 3A). Classification error for a
$5 \mathrm{MC}$ classifier for subject $\mathrm{TH} 2$ was low across all electrode configurations, but marginally $(1.2 \%)$ higher for the grid compared to the targeted configurations.

We also evaluated the classification error for 7 and $9 \mathrm{MC}$ classifiers, for both subjects. The results were consistent for both subjects, indicating reduced classification error when electrodes are arranged in a grid as compared to direct control sites. Classification error was highest when 4 bipolar electrode pairs located at direct control sites were used. Classification error was slightly lower when 8 instead of the 4 bipolar electrodes were used and the error was lowest when grid electrode configuration was used by the classifiers (Fig. 3).

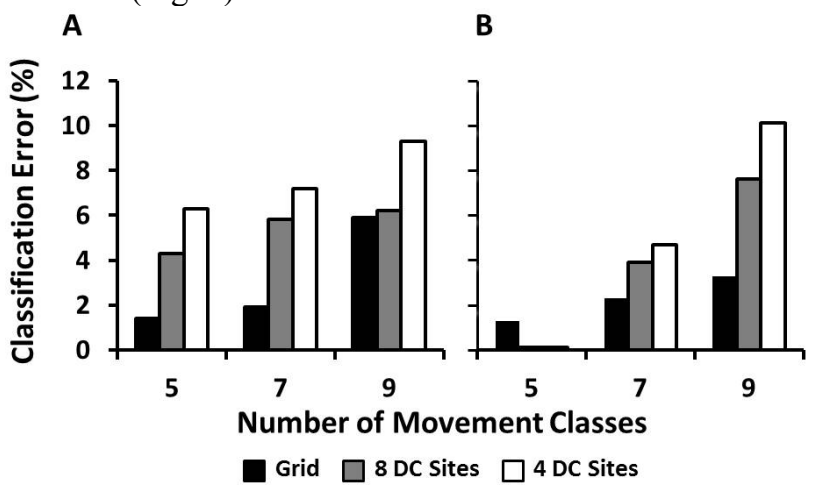

Fig.3 Real-time classification error of the 5, 7 and 9 MC classifiers. Data is shown for subject $\mathrm{TH} 1$ (A) and $\mathrm{TH} 2$ (B) and for both experimental conditions. Black bars represent classification error when 15 EMG electrodes were arranged in a grid. TH1 grid configuration errors for 5,7 and $9 \mathrm{MC}$ classifiers were: $1.4 \%, 1.9 \%$ and $5.9 \%$ respectively. $\mathrm{TH} 2$ errors were: $1.3 \%, 2.3 \%$ and $3.3 \%$, respectively. Grey bars indicate classification errors from 8 bipolar electrodes placed at 4 direct control sites. TH1 direct control site errors for 5,7 and $9 \mathrm{MC}$ classifiers were: $4.3 \%, 5.8 \%$ and $6.2 \%$ respectively. $\mathrm{TH} 2$ errors were: $0.1 \%, 3.9 \%$ and $7.6 \%$, respectively. White bars show classification errors from using 4 of the 8 bipolar electrodes placed at the direct control sites. TH1 errors for 5,7 and $9 \mathrm{MC}$ classifiers were: $6.3 \%, 7.2 \%$ and $9.3 \%$ respectively. $\mathrm{TH} 2$ errors were: $0.1 \%, 4.7 \%$ and $10.1 \%$, respectively.

Although a 15 electrode grid minimizes classification error of an LDA classifier for 5, 7 and 9 classes of movement, the additional number of channels in the grid imposes a higher computational cost than the 8 or 4 bipolar electrodes at the direct control sites. Computational power of the currently available microprocessors likely alleviates this concern. Nonetheless, we performed a channel reduction analysis to evaluate the relationship between classification error and the number of electrodes used by the classifier. The results of this analysis demonstrated that classification error plateaus at a subset of 7 channels for the three types of classifiers, for both subjects (Fig. 4). The subset of 7 channels was composed of both cross-paired as well as longitudinally paired electrodes (Fig. 1B). 


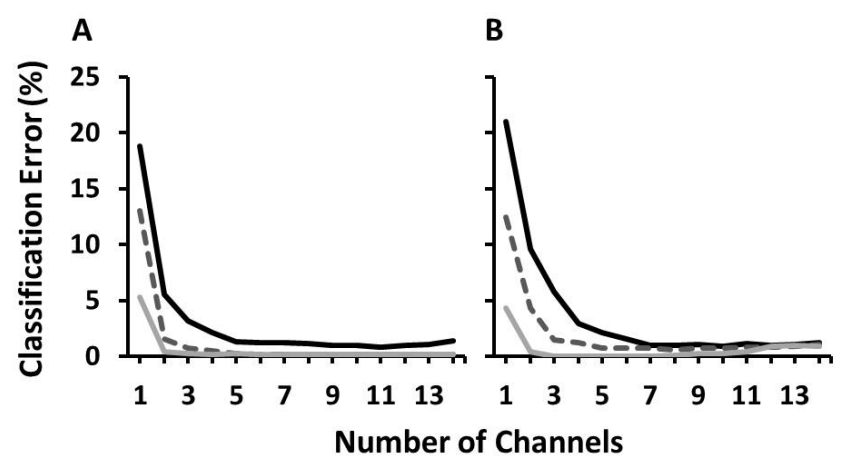

Fig.4 Classification error of the 9, 7 and $5 \mathrm{MC}$ classifiers as a function of the number of channels used for both subjects TH1 (A) and TH2 (B).Demonstrated classification error is computed based on the optimal subset of channels, for channel subsets ranging in size from 1 to 14 . Classification errors of the $9 \mathrm{MC}$ classifier are shown in solid black line and the $7 \mathrm{MC}$ classifier are shown in punctuated dark-grey line. The solid light grey line demonstrated the classification errors of a $5 \mathrm{MC}$ classifier.

\section{DISCUSSION}

This pilot study demonstrated that pattern recognition control does not require precise placement of electrodes over the conventional, "direct" control muscle sites in TMR patients. Our results demonstrate that 15 electrodes, evenly distributed along and around the residual limb, will yield control accuracy and motion completion times that are on par or superior to what can be achieved via precise electrode placement over direct control sites. Furthermore, our results also suggest that the number of channels used may be dynamically reduced using an automated algorithm to 7 electrodes, which decreases classification error and computational cost. Such a technique could be easily incorporated into a myoelectric controller to optimize the use of a 15 electrode grid. These are important results, as the use of an electrode grid is substantially easier to implement clinically than targeted placement of electrodes.

The results of this study differ from those presented previously [15], which indicated benefit of using electrodes located directly over the TMR control sites. The interelectrode distances used in [10] $(15-20 \mathrm{~mm})$ were smaller than those used in this study (30-100 mm). We have shown previously that larger inter-electrode distances significantly reduces classification accuracies [16] presumably because more of the underlying muscles are sampled. This, along with investigating fewer motions ( 9 movement classes as opposed to 16) may have led to the discrepancy in the findings.

Notable limitations of this pilot study include the small subject sample size, small number of movement trials and controllability assessment limited to 5 movement classes. Future work will focus on collecting a larger pool of classifier performance data for a broader set of movements, including simultaneous movement sequences. Analogous to the grid channel reduction analysis presented in the current study, grid feature reduction analysis will also be performed to further evaluate the means of reducing classification error, computation cost, and optimize grid size and configuration.

Our findings have significant implications as they offer a practical, cost effective means to increase transhumeral amputee patient access to myoelectric prostheses by greatly reducing the complexity and costs associated with fitting amputees with myoelectric devices. Our findings also indicate that a grid electrode arrangement also has the potential to offer greatest possible classification accuracies through utilization of channel optimization algorithms.

\section{ACKNOWLEDGMENT}

The authors would like to thank Robert Parks, Tom Idstein, Connor Griffith and Todd Kuiken for providing project support and guidance.

\section{REFERENCES}

[1] K. Ziegler-Graham, E. J. MacKenzie, P. L. Ephraim, T. G. Travison, and R. Brookmeyer, "Estimating the prevalence of limb loss in the United States: 2005 to 2050," Archives of Physical Medicine and Rehabilitation, vol. 89, pp. 422-429, Mar 2008.

[2] M. Zecca, S. Micera, M. C. Carrozza, and P. Dario, "Control of multifunctional prosthetic hands by processing the electromyographic signal," Critical Reviews in Biomedical Engineering, vol. 40, pp. 459485, 2002.

[3] P. Parker, K. Englehart, and B. Hudgins, "Myoelectric signal processing for control of powered limb prostheses," Journal of Electromyography and Kinesiology, vol. 16, pp. 541-548, DEC 2006.

[4] T. A. Kuiken, G. Li, B. A. Lock, R. D. Lipschutz, L. A. Miller, K. A. Stubblefield, and K. B. Englehart, "Targeted muscle reinnervation for real-time myoelectric control of multifunction artificial arms," JAMA, vol. 301, pp. 619-28, Feb 112009.

[5] C. Alstrom, P. Herberts, and L. Korner, "Experience with Swedish Multifunctional Prosthetic Hands Controlled by Pattern Recognition of Multiple Myoelectric Signals.," International Orthopedics, vol. 5, pp. $15-21,1981$.

[6] K. Englehart and B. Hudgins, "A robust, real-time control scheme for multifunction myoelectric control," IEEE Transactions on Biomedical Engineering, vol. 50, pp. 848-54, 2003.

[7] L. Hargrove, K. Englehart, and B. Hudgins, "A Comparison of Surface and Intramuscular Myoelectric Signal Classification," IEEE Transactions on Biomedical Engineering, vol. 54, pp. 847-853, 2007.

[8] T. R. Farrell and R. F. Weir, "A comparison of the effects of electrode implantation and targeting on pattern classification accuracy for prosthesis control," IEEE Trans Biomed Eng, vol. 55, pp. 2198-211, Sep 2008.

[9] H. Daley, K. Englehart, L. Hargrove, and U. Kuruganti, "High Density Electromyography Data of Normally Limbed and Transradial Amputee Subjects for Multifunction Prosthetic Control," Journal of Electromyography and Kinesiology, vol. In Press, 2012.

[10]H. Huang, P. Zhou, G. Li, and T. A. Kuiken, "An Analysis of EMG Electrode Configuration for Targeted Muscle Reinnervation Based Neural Machine Interface," IEEE Transactions on Neural Systems and Rehabilitation Engineering, vol. 16, pp. 37-45, 2008.

[11]L. H. Smith, L. Hargrove, B. A. Lock, and T. Kuiken, "Determining the optimal window length for pattern recognition-based myoelectric control: balancing the competing effects of classification error and controller delay," IEEE Transactions on Neural Systems and Rehabilitation Engineering, vol. 19, pp. 186-192, 2011.

[12]Y. H. Huang, K. B. Englehart, B. Hudgins, and A. D. C. Chan, "A Gaussian mixture model based classification scheme for myoelectric control of powered upper limb prostheses," IEEE Transactions on Biomedical Engineering, vol. 52, pp. 1801-1811, 2005.

[13]A. Simon, L. Hargrove, B. A. Lock, and T. Kuiken, "Target Achievement Control Test: Evaluating real-time myoelectric patternrecognition control of multifunctional upper-limb prostheses " Journal 
of Rehabilitation Research and Development, vol. 18, pp. 619-628,

2011.

[14]A. Simon, L. Hargrove, B. A. Lock, and T. Kuiken, "A decision-based velocity ramp for minimizing the effect of misclassifications during real-time pattern recognition control," IEEE Transactions on Biomedical Engineering, vol. 58, pp. 2360 - 2368, 2011.

[15]H. Huang, T. Kuiken, and R. Lipschutz, "A Strategy for Identifying Locomotion Modes using Surface Electromyography," IEEE Trans Biomed Eng, vol. 56, pp. 65-73, Jan 20092009.

[16]A. Young, L. Hargrove, and T. Kuiken, "Improving Myoelectric Pattern Recognition Robustness to Electrode Shift by Changing Interelectrode Distance and Electrode Configuration," IEEE Transactions on Biomedical Engineering, vol. 59, pp. 645-652, 2012. 